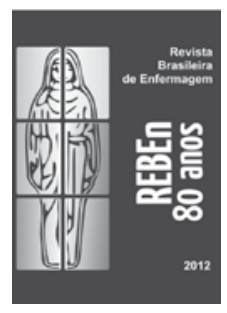

\title{
Departamento Científico de Enfermagem Gerontológica da Associação Brasileira de Enfermagem
}

\author{
Scientific Department of Gerontological Nursing of the Brazilian Nursing Association \\ Departamento Científico de Enfermería Gerontológica de la Asociación Brasileña de Enfermería
}

\begin{abstract}
Angela Maria Alvarez', Annelita Almeida de Oliveira Reiners", Sandra Helena Isse Polaro'"I, Lucia Hisako Takase Gonçalvesiv ${ }^{\mathrm{I}}$, Célia Pereira Caldas" ${ }^{\mathrm{v}}$, Margarita Ana Rubin Unicovsky ${ }^{\mathrm{V}}$, Maria Célia de Freitas ${ }^{\mathrm{VII}}$

'Universidade Federal de Santa Catarina. Florianópolis-SC, Brasil. Associação Brasileira de Enfermagem, Departamento Científico de Enfermagem Gerontológica. Brasília-DF, Brasil.

"Universidade Federal do Mato Grosso. Cuiabá-MT, Brasil. Associação Brasileira de Enfermagem,

Departamento Científico de Enfermagem Gerontológica. Brasília-DF, Brasil.

III Universidade Federal do Pará. Belém-PA, Brasil. Associação Brasileira de Enfermagem,

Departamento Científico de Enfermagem Gerontológica. Brasília-DF, Brasil.

Iv Universidade Federal do Pará, Instituto de Ciências da Saúde, Programa de Mestrado em Enfermagem.Belém-PA, Brasil.

${ }^{\checkmark}$ Universidade do Estado do Rio de Janeiro. Rio de Janeiro-RJ, Brasil. Associação Brasileira de Enfermagem,

Departamento Científico de Enfermagem Gerontológica. Brasília-DF, Brasil.

V' Universidade Federal do Rio Grande do Sul. Porto Alegre-RS, Brasil. Associação Brasileira de Enfermagem,

Departamento Científico de Enfermagem Gerontológica. Brasília-DF, Brasil.

VII Universidade Estadual do Ceará. Fortaleza-CE, Brasil. Associação Brasileira de Enfermagem,

Departamento Científico de Enfermagem Gerontológica. Brasília-DF, Brasil.
\end{abstract}

Submissão: 05-09-2013 Aprovação: 05-09-2013

\section{RESUMO}

Objetivou-se refletir sobre a criação do Departamento Científico de Enfermagem Gerontológica no âmbito da Associação Brasileira de Enfermagem, considerando o contexto do envelhecimento populacional e o desenvolvimento e consolidação da especialidade no país. No processo reflexivo contou-se com a análise histórica da literatura revista e dos relatos dos autores. Os resultados mostraram o movimento de enfermeiras nas últimas décadas para produzir conhecimento, assim como sua incorporação pela entidade e o empenho de seus membros para alcançar as metas propostas para o Departamento Científico de Enfermagem Gerontológica. Conclui-se que sua criação representa um marco para a organização política e científica dos profissionais de enfermagem que buscam qualificar-se para um cuidado autônomo e competente às pessoas idosas e suas famílias.

Descritores: Enfermagem; Saúde do Idoso; Cuidados de Enfermagem.

\begin{abstract}
This paper aimed to consider the creation of the Scientific Department of Gerontological Nursing within the Brazilian Nursing Association, considering the context of population ageing, and the development and consolidation of this specialty in the country. In the reflexive process, it was possible to rely on the historical analysis of the revised literature and authors' reports. The results showed the movement of nurses, in recent decades, to the production of knowledge, as well as their incorporation by the entity, and the commitment of its members to achieve the goals proposed to the Scientific Department of Gerontological Nursing. It is concluded that its creation represents a milestone to the scientific and political organization of the nursing professionals who seek to qualify themselves for an autonomous and competent care of the elderly and their families.
\end{abstract}

Key words: Nursing; Elderly Health; Nursing Care. 


\section{RESUMEN}

El objetivo fue discurrir sobre la creación del Departamento Científico de Enfermería Gerontológica en el ámbito de la Asociación Brasileña de Enfermería, considerando el contexto de envejecimiento poblacional, y el desarrollo y consolidación de la especialidad en el país. En el proceso reflexivo se contó con el análisis histórico de la literatura revisada y los relatos de los autores. Los resultados mostraron el movimiento de enfermeras, en las últimas décadas, para la producción de conocimiento en gerontología, así como su incorporación por la entidad y el empeño de sus miembros para el alcance de las metas propuestas para el Departamento Científico de Enfermería Gerontológica. Se concluye que su creación representa un marco para la organización política y científica de los profesionales de enfermería que buscan calificarse para un cuidado autónomo y competente a los ancianos y sus familias.

Descriptores: Enfermería; Salud de los Ancianos; Atención de Enfermería.

\section{AUTOR CORRESPONDENTE Angela Maria Alvarez E-mail: angela.alvarez@ufsc.br}

\section{INTRODUÇÃO}

O envelhecimento populacional é importante fenômeno observado na dinâmica demográfica mundial atual. Nos países desenvolvidos, esse processo já é parte de sua realidade há décadas. Neles, construíram-se alicerces de uma sociedade mais inclusiva e mais adequada à população idosa. Hoje, esse envelhecimento também está em curso nos países em desenvolvimento, nos quais, apesar dos avanços normativos e institucionais, muito ainda precisa ser feito para que a velhice seja associada a uma vida ativa e saudável amparada por políticas e programas condizentes ${ }^{(1)}$.

O crescimento da população de idosos na composição da população geral é um fenômeno mundial e está ocorrendo a uma velocidade sem precedentes. Em 1950 existiam cerca de 240 milhões de idosos no mundo; em 1998, após quase cinco décadas, chegava-se a 579 milhões de pessoas, um crescimento de quase oito milhões de idosos a cada ano. As projeções indicam que em 2050 a população idosa será de 1,9 biIhão de pessoas, igualando-se à população infanto-juvenil ${ }^{(2-3)}$.

O envelhecimento da população brasileira nas duas últimas décadas trouxe implicações substanciais para o cuidado em enfermagem, pois os 20.590 .597 milhões de pessoas idosas representam $10,79 \%$ do total da população. Mesmo considerando que a maioria dos idosos $(55,12 \%)$ encontra-se entre os mais jovens, entre 60 e 69 anos, há uma grande preocupação com o subgrupo dos idosos de 80 anos ou mais. Nessa faixa etária surgem demandas de cuidados prolongados e uma redução da oferta de cuidadores familiares, com a tendência de nas próximas décadas aumentar significativamente esse grupo, que já representa $14,25 \%$ da população ${ }^{(1,4)}$.

Isso exige maiores e intensivos esforços na revisão de políticas, programas e ações com vista ao envelhecimento saudável da população, contemplando a promoção e a proteção da velhice no cuidado a pessoa idosa numa perspectiva de valorização do ser humano. Tais questões afetam diretamente a Enfermagem. Especialidade emergente no Brasil, a Enfermagem Gerontológica vem sendo impulsionada a dar respostas concretas para o cuidado ao idoso e à família que toma para si essa responsabilidade sem se descuidar da qualidade do cuidado prestado em instituições assistenciais.
Historicamente, o interesse da Enfermagem brasileira no estudo do cuidado do envelhecimento humano está presente nas publicações a partir da década de 80 , ainda que timidamente, mas de modo contínuo. Já no ensino da graduação esse despertar se mostra nas discussões das Diretrizes Curriculares Nacionais do Curso de Graduação em Enfermagem aprovadas pelo Conselho Nacional de Educação(5) , nas quais estão previstos projetos pedagógicos para atender ao perfil demográfico e epidemiológico, o que sugere a inclusão do estudo sobre o envelhecimento humano nos diferentes níveis de ensino ${ }^{(6)}$.

O método histórico foi usado para registrar e analisar toda uma trajetória da evolução das produções e seus produtores da especialidade, revelando o estado da arte da Enfermagem Gerontológica no Brasil. Entre os dados levantados, registrou-se que as produções de pesquisa, no total de 473 , são oriundas de 39 grupos de pesquisa sobre estudo do envelhecimento ou de grupos com linhas de pesquisa sobre envelhecimento dos programas de pós-graduação em enfermagem do país. Os grupos de pesquisa foram sendo criados ao longo do tempo, denotando que o escopo da produção científica em Enfermagem Gerontológica e o número crescente de grupos de estudo refletem os avanços teórico-metodológicos desse conhecimento especializado ${ }^{(7-9)}$.

Especificamente na Revista Brasileira de Enfermagem (RE$\left.B n^{\circ}\right)$, uma pesquisa buscando identificar artigos da Enfermagem sobre envelhecimento, no período de 2000 a 2006, encontrou 18 artigos científicos ali publicados sobre envelhecimento no período especificado. As autoras enfatizam que, embora se mostre incipiente, a produção é de grande relevância para o período ${ }^{(10)}$.

Em levantamento realizado na REBEn ${ }^{\circledast}$ para o presente estudo, identificou-se, no período de cinco anos - 2007 a 2012 - o significativo salto para 57 artigos científicos, aumento importante da produção na área, considerando-se que o dado se refere apenas a um periódico de enfermagem do país. Somados os períodos estudados, em 12 anos encontramos a produção de 75 artigos científicos publicados na REBEn ${ }^{\oplus}$. Tais estudos exploram, em sua maioria, temas como: avaliação clínica da pessoa idosa e o cuidado de enfermagem; a família cuidadora; o cuidado ao idoso e o cuidador; o ensino da Enfermagem Gerontológica e o idoso na instituição de longa permanência. 
O interesse da Enfermagem pela área do envelhecimento ocorreu também em conjunto com outros grupos profissionais, como da medicina, que expandia a especialidade da geriatria; do serviço social, que discutia requerimentos de políticas públicas específicas para a população idosa; e, mais adiante, houve a incorporação da educação física, da psicologia e de outras disciplinas que concorreriam para a atenção interdisciplinar com enfoque multidimensional dos clientes idosos. $\mathrm{O}$ crescimento da Gerontologia no país tem-se dado muito pela participação do conjunto dos profissionais especialistas na Sociedade Brasileira de Geriatria e Gerontologia, que contribui para promover o desenvolvimento da área, realizando eventos de várias naturezas e capacitando seus associados ${ }^{(6)}$.

A Enfermagem, de forma crescente nas três últimas décadas, busca o desenvolvimento da Enfermagem Gerontológica, iniciando ações educacionais e produzindo conhecimentos na área. Em outro estudo sobre análise da autonomia profissional da enfermeira gerontóloga junto ao cliente idoso no contexto de trabalho em equipe interprofissional com enfoque interdisciplinar, as respostas obtidas recaíram sobre as categorias: conhecimento, postura profissional, aplicação da sistematização da assistência e delimitação do papel da enfermagem na equipe multiprofissional. Houve reconhecimento do espaço para atuação autônoma, embora sua concretude esteja em processo de construção, intrinsecamente atrelada ao conhecimento especializado e à formação profissional competente. E o poder profissional da enfermeira gerontóloga se fortaleceu na medida da robustez dos conhecimentos construídos ${ }^{(7)}$.

Nos grupos de pesquisa e cursos de pós-graduação registra-se um movimento na trajetória histórica de busca por avanços da especialidade por meio de um evento científico regular, a Jornada Brasileira de Enfermagem Geriátrica e Gerontológica. A primeira ocorreu em 1996, em Florianópolis-SC, idealizada pelo Grupo de Estudo sobre Saúde de Pessoas Idosas - GESPI do Programa de Pós-Graduação de Enfermagem, e apoiada pelo Núcleo Interdisciplinar de Assistência, Ensino e Pesquisa Geriátrica do Hospital Universitário e pelo Núcleo de Estudos da Terceira Idade, todos da Universidade Federal de Santa Catarina e da Associação Brasileira de Enfermagem - Seção Santa Catarina. A temática desenvolvida nessa Jornada referiu-se a ciência, tecnologia, arte, ética e estética da Enfermagem, confluindo para o envelhecimento humano. Desde então, esse evento passou a ser realizado bienalmente e em diferentes regiões do país, organizado por outros grupos de estudo e por docentes, pesquisadores e enfermeiros ligados à área. A II Jornada, em Foz do Iguaçu-PR, teve como temática de discussão a especificidade da Enfermagem Gerontológica. Na III Jornada, em Salvador-BA, discutiu-se a formação de recursos humanos e a expansão da especialidade. Na IV Jornada, em Santos-SP, discutiu-se a questão dos cuidadores leigos de idosos e suas implicações para a Enfermagem. A V Jornada voltou a ser realizada em Florianópolis-SC, tendo como tendo como O cuidado do idoso contemporâneo. Pela primeira vez foi realizada por uma Seção da ABEn, a Seção Santa Catarina, com o apoio do mesmo grupo da Jornada inicial, agora ampliado e já bem mais experiente. Os organizadores e participantes dessa Jornada protagonizaram um movimento para criar, no âmbito da ABEn Nacional, um grupo de interesse em Enfermagem Gerontológica. Dali, seguiram-se reuniões do grupo de interesse nos muitos eventos da ABEn que ocorriam em todo o país, para propagar entre os abenistas a necessidade de organizar a especialidade. A VI Jornada ocorreu em Ribeirão Preto-SP, onde se discutiu a Fragilidade da Vida e os Desafios da Longevidade. Já a VII Jornada foi organizada pelas enfermeiras da ABEn Seção Rio Grande do Sul e ocorreu em Porto Alegre, na qual se discutiram os desafios da formação especializada de enfermagem na atenção ao idoso. Em 2010, a VIII Jornada é organizada pelas enfermeiras da ABEn - Seção Rio de Janeiro para discutir os Desafios da enfermagem na assistência integral do idoso. E, por fim, em 2012 a IX Jornada foi realizada em Fortaleza-CE, já sob a coordenação do Departamento Científico de Enfermagem Gerontológica da ABEn e realizada pela ABEn Seção Ceará.

Esse grupo de enfermeiros e estudantes, que no decorrer dos anos se congregaram em torno das jornadas, por ocasião do $15^{\circ}$ Seminário Nacional de Pesquisa em Enfermagem, no Rio de Janeiro-RJ, em junho de 2009, propuseram formalmente à Diretoria da ABEn Nacional a criação do grupo de Enfermagem Gerontológica. A proposta foi analisada e acatada pelo ConseIho Nacional da ABEn (CONABEn) em reunião na cidade de Fortaleza-CE, durante o $60^{\circ} \mathrm{CBEn}$. Na sequência, o Departamento Científico de Enfermagem Gerontológica (DEGER) foi criado pela Resolução CONABEn No 001/2009 ${ }^{(11)}$.

$\mathrm{Na}$ época, a mensagem da representante no CONABEn transmitida ao grupo de interesse da Enfermagem Gerontológica foi recebida com justa alegria e propósito de continuar lutando por aquele ideal e de congregar enfermeiros da especialidade no seio da ABEn. Eis o teor de duas comunicações trocadas entre membros do grupo:

[...] O Departamento Científico de Enfermagem Gerontologia foi aprovado no CONABEn, no dia de hoje, às 22h30min. Amanhã passará na Assembléia Nacional de Delegados - instância máxima da ABEn. Encaminho um documento preliminar que montei para defesa no CONABEn. Não está bom. Será revisado para ser transformado em Resolução. A aceitação de todos foi muito legal. Um momento de alegria foi quando pude falar um pouco da nossa história e de vocês. Um abraço a todas. (Angela)

[...] Queridas amigas, Foi com muita emoção que recebi a notícia da aprovação do Departamento no CONABEn. Um grande sonho que se torna realidade. O melhor de tudo é a consciência de que estamos cumprindo o nosso papel na história da Enfermagem. É muita felicidade! Vocês são as grandes guerreiras precursoras desta causa. Vocês me motivaram a lutar pela instituição da Enfermagem Gerontológica em nosso país. Amo vocês! (Célia)

No início do ano seguinte, em 07 de fevereiro de 2010, o Regimento Interno do Departamento Científico de Enfermagem Gerontológica ${ }^{(12)}$ foi aprovado pelo CONABEn, definindo sua forma de organização como órgão assessor da diretoria da ABEn Nacional e suas competências: 
1. Elaborar plano de trabalho e submetê-lo à aprovação da Diretoria da ABEn Nacional.

2. Promover, bienalmente, a Jornada Brasileira de Enfermagem Geriátrica e Gerontológica (JBEGG).

3. Realizar, nas seções, regionais e núcleos da ABEn, cursos de aperfeiçoamento e treinamento para Enfermeiros e Técnicos de Enfermagem, que favoreçam a capacitação para o cuidado de pessoas idosas.

4. Propor medidas necessárias à defesa e consolidação do trabalho em Enfermagem Gerontológica.

5. Representar a ABEn nos fóruns de Geriatria, de Gerontologia e de áreas afins e de interesse.

6. Participar efetivamente dos movimentos em defesa da cidadania da pessoa idosa.

7. Prestar consultoria técnica, quando solicitada, no planejamento de projetos, programas e serviços nas áreas de saúde física, mental e social da pessoa idosa.

8. Produzir e divulgar, em instrumento próprio, resultados de trabalhos e estudos de interesse para as áreas de saúde física, mental e social da pessoa idosa.

9. Colaborar na manutenção de um centro de informações acadêmicas e científicas para fomentar estudos pertinentes à Enfermagem Gerontológica em parceria com CEPEN/ABEn.

10. Participar, com a ABEn, na organização do processo de concessão de título de Especialista em Enfermagem Gerontológica.

11. Apresentar relatório anual de trabalho à Diretoria da $A B E n$.

Para compor o Departamento, esperou-se pela realização da VIII Jornada Brasileira de Enfermagem Geriátrica e Gerontológica, que ocorreria em abril de 2010, para que o grupo de interesse apresentasse à Diretoria da ABEn Nacional os nomes das enfermeiras escolhidas com base no que fora definido no regimento interno (cinco enfermeiros especialistas, com comprovada experiência na área, representando as cinco regiões geográficas do país e um representante da Diretoria da ABEn Nacional). O processo ocorreu numa reunião ampliada e convocada para esse fim. Dessa forma, o Núcleo Coordenador do Departamento Científico de Enfermagem Gerontológica foi constituído pela Portaria $\mathrm{n}^{\circ} 002 / 2010^{(13)}$ com os seguintes integrantes: Rosalina Aratami Sudo (Diretora Científico-Cultural da ABEn Nacional); Angela Maria Alvarez (representante da Região Sul); Adélla Yaeko Kyosen Nakatami (representante da Região Centro Oeste); Célia Pereira Caldas (representante da Região Sudeste); Maria Célia de Freitas (representante da Região Nordeste) e Sandra Helena Isse Polaro (representante da Região Norte). A coordenação desse grupo ficou ao encargo de Angela Maria Alvarez, designada na mesma Portaria.

Naquele mesmo ano, o DEGER, com apoio da Presidente da ABEn, Maria Goretti David Lopes, encaminhou ao Ministério da Saúde, para financiamento, o Projeto Capacitação de Enfermeiros da Estratégia de Saúde da Família para a Atenção à Saúde do Idoso. Sua fundamentação baseou-se nos marcos teóricos conceituais da Política Nacional de Saúde do Idoso, da Atenção Básica e da Promoção de Saúde. Os objetivos foram: capacitar enfermeiros da Estratégia de Saúde da Família para a atenção à saúde do idoso com vistas à promoção do envelhecimento ativo, recuperação da saúde e a prevenção de agravos; criação e organização do Departamento Científico de Enfermagem Gerontológica na Seção da ABEn onde a capacitação seria realizada. $O$ projeto foi aprovado com recursos de noventa e dois mil reais e contrapartida da ABEn Nacional de oito mil reais para realizar oficinas nas diferentes regiões do país. Dessa forma, a meta principal do DEGER passou a ser a capacitação de enfermeiros para a atenção à saúde do idoso na Estratégia de Saúde da Família.

Um revés: os recursos financeiros do Ministério da Saúde para o projeto, aprovados no ano de 2010, só seriam liberados em setembro de 2012. Isso não foi empecilho para a realização de reuniões e desenvolvimento de oficinas nos espaços dos eventos promovidos pela ABEn, numa atitude proativa dos membros do DEGER e com a colaboração voluntária de enfermeiros ligados à causa, como Lúcia Hisako Takase Gonçalves, Maria do Livramento Fortes Figueiredo e Gleide Magali Pinheiro Lemos.

No ano de 2011, um acordo com a nova diretoria da ABEn Nacional Gestão 2010-2013 permitiria que os mesmos integrantes continuassem a desenvolver o plano de trabalho traçado ainda no ano de 2010, pois o grupo recém iniciara a coordenação das atividades do Departamento. Mesmo diante dessa perspectiva houve a necessidade de recomposição de parte do grupo, devido à mudança da representante da Diretoria da ABEn e do impedimento de uma das integrantes. Assim, para a gestão 2010 - 2013, nova Portaria da Presidente da ABEn, Ivone Evangelista Cabral, designou Margarita Ana Rubin Unicowisky, Angela Maria Alvarez, Célia Pereira Caldas, Maria Célia de Freitas, Sandra Helena Isse Polaro e Annelita Almeida de Oliveira Reiners. A coordenação permaneceu sob a liderança de Angela Maria Alvarez, conforme a Portaria 003/ 2011 ${ }^{(14)}$.

Em 2011 iniciou-se a preparação da IX Jornada Brasileira de Enfermagem Geriátrica e Gerontológica a ser realizada pela ABEn Seção Ceará, sob a coordenação de Maria Célia de Freitas, que se realizaria nos dias 19, 20 e 21 de setembro de 2012, com a programação científica pautando-se pelo tema central $A$ interface do cuidado de enfermagem com as políticas de atenção ao idoso, traduzindo a preocupação da entidade com a instrumentalização da equipe de enfermagem para promover um cuidado técnico-científico, ético e solidário, diante da mudança do perfil epidemiológico da população brasileira e do aumento crescente da expectativa de vida.

Esse evento deu continuidade, agora oficialmente como evento da ABEn Nacional, às edições anteriores da jornada realizadas pelo grupo de interesse e resultou na carta de Fortaleza, que foi encaminhada às autoridades, destacando a preocupação dos participantes da jornada em relação a regulamentar a profissionalização do cuidador de idosos. O tema suscitou debate e reflexões que mereceram a atenção dos profissionais de enfermagem, das autoridades e da sociedade em geral. A carta segue solicitando a participação da Enfermagem no debate e destaca o esforço pelo histórico da enfermagem em qualificar os membros de sua equipe e a necessidade de atenção digna e qualificada às pessoas idosas ${ }^{(15-16)}$.

No segundo semestre de 2012 e primeiro de 2013, as atividades do DEGER foram intensificadas na capacitação 
de enfermeiros da ESF e criação dos Departamentos Científicos de Enfermagem Gerontológica nas diferentes Seções da ABEn. Duas oficinas de Capacitação de Enfermeiros da Estratégia Saúde da Família na atenção à saúde do idoso foram realizadas. Uma em Porto Alegre em agosto de 2012 e a segunda em Cuiabá, em maio de 2013. Cinco oficinas de Capacitação de Enfermeiros como agentes multiplicadores da atenção à pessoa idosa foram desenvolvidas no ano de 2013, no Rio de Janeiro, em Florianópolis, em Fortaleza, em Belém e, por fim, em Manaus. Duas oficinas para desenvolver e fortalecer os departamento científicos de Enfermagem Gerontológica nas seções da ABEn foram realizadas em Brasília. Dessa forma, participaram das oficinas 756 enfermeiros e foram organizados os Departamentos em seis Seções da ABEn: Rio Grande do Sul, Rio de Janeiro, Santa Catarina, Ceará, Pará e Mato Grosso.

\section{CONCLUSÃO}

A criação do DEGER na estrutura da ABEn Nacional e em algumas de suas Seções representa um marco para a organização política e científica dos profissionais de Enfermagem que buscam qualificar-se rumo a um cuidado autônomo e competente dirigido às pessoas idosas e suas famílias. Ao mesmo tempo, constitui-se num importante passo na representatividade da área, para o debate da política de formação no contexto da própria Enfermagem e de políticas públicas de atenção às pessoas idosa. Necessário se faz continuar a expandir suas ações congregando os associados, profissionais (enfermeiros assistenciais, docentes e pesquisadores), assim como os estudantes, para o fortalecimento da especialidade e o incentivo à consolidação da área nos projetos pedagógicos dos cursos de graduação e de ensino profissional em enfermagem.

\section{REFERÊNCIAS}

1. Camarano AA, organizador. Cuidados de longa duração para a população idosa: um novo risco social a ser assumido? Rio de Janeiro: IPEA; 2010.

2. Instituto Brasileiro de Geografia e Estatística. Perfil dos idosos responsáveis por domicílios no Brasil 2000/IBGE. Rio de Janeiro: Departamento de População e Indicadores Sociais; 2002.

3. Naciones Unidas. Departamento de Asuntos Económicos y Sociales El informe - Estudio Económico y Social Mundial 2007 - El desarrollo en um mundo que envejece. Reseña. Naciones Unidas: Nueva York; 2007.

4. Instituto Brasileiro de Geografia e Estatística [homepage na internet]. Sistema IBGE de Recuperação Automatica (SIDRA). Censo Demográfico 2010 - Tabela 1378: População residente, por situação de domicílio, sexo e idade, segundo condição no domicílio e compar.ilhamento da responsabilidade pelo domicílio. [Acesso em 03 set 2013]. Disponível: http://www.sidra.ibge.gov.br/bda/tabela/protabl.asp?c $=1378 \& \mathrm{z}=\mathrm{t} \& \mathrm{O}=25 \& \mathrm{i}=\mathrm{P}$.

5. Conselho Nacional de Educação - Câmara de Educação Superior (Brasil) Resolução No 3, de 7 de novembro de 2001. Dispõe sobre as Diretrizes Curriculares Nacionais do Curso de Graduação em Enfermagem. Diário Oficial da União de 3 de out 2001, Seção 1E, p. 131.

6. Gonçalves LHT, Alvarez AM. O cuidado na enfermagem gerontogeriátrica: conceito e prática. In: Freitas EV, organizador. Tratado de geriatria e gerontologia. 2. ed. Rio de Janeiro: Guanabara Koogan; 2006. p.1110-16.

7. Kletemberg DF, Padilha MI Borestein MS, Goncalves LHT, Alvarez AM, Ferreira AC. A construção histórica do conhecimento da enfermagem gerontológica no Brasil. Esc Anna Nery Rev Enferm 2010;14(4):787-796.

8. Kletemberg DF, Padilha MI. A autonomia da enfermagem gerontológica no Brasil, segundo as pioneiras (19701996) Texto \& Contexto Enferm 2011;20(4):709-16.
9. Kletemberg DF, Padilha MI. Enfermagem gerontológica: a produção do conhecimento na profissão (1970-1996). Rev Gaúcha Enferm 2013;34(1):86-93.

10. Rocha FCV, Brito CMS, Luz MHBA, Figueiredo MLF. Análise da produção científica sobre o idoso na REBEn. Rev Bras Enferm 2007;60(4):449-451.

11. Associação Brasileira de Enfermagem. Resolução CONABEn n. ${ }^{\circ}$ 01/2009, de 15 de dezembro de 2009. Dispõe sobre a criação do Departamento Científico de Enfermagem Gerontológica [resolução na internet]. 2009 [acesso em 04 set 2013]. Disponível em: http://www.abennacional. org.br/index.php? path $=260$.

12. Associação Brasileira de Enfermagem. Departamento científico de enfermagem gerontológica. Regimento Interno. Aprovado em 07 de fevereiro de 2010 [regimento interno na internet]. 2010 [acesso em 3 set de 2013]. Disponível em: http://www.abennacional.org.br/index. php? path $=260$.

13. Associação Brasileira de Enfermagem. Portaria n. ${ }^{\circ}$ 02/2010, de 16 de junho de 2010. Dispõe sobre a Nomeação dos Componentes do Departamento Científico de Enfermagem Gerontológica. [portaria na internet]. 2010 [Acesso em 04 set 2013]. Disponível em: http://www. abennacional.org.br/index.php?path $=260$.

14. Associação Brasileira de Enfermagem. Portaria n. ${ }^{\circ}$ 03/2011, de 09 de fevereiro de 2011. Dispõe sobre a Nomeação dos Componentes do Departamento Científico de Enfermagem Gerontológica. [portaria na internet]. 2010 [acesso em 04 set 2013]. Disponível em: http:// www.abennacional.org.br/index.php?path $=260$.

15. Alvarez AM, Goncalves, LHT. Enfermagem e o cuidado ao idoso no domicilio. Rev Bras Enferm 2012; 65(5):715-716.

16. Motta LB da, Aguiar AC de, Caldas, CP. Estratégia Saúde da Família e a atenção ao idoso: experiências em três municípios brasileiros. Cad Saúde Pública 2011;27(4):779-86. 